\title{
COMPUTATION OF QUANTUM FLUCTUATIONS OF QUARK FIELDS IN AN ARBITRARY YANG-MILLS INSTANTON BACKGROUND
}

\author{
B. BERG \\ II. Institut für Theoretische Physik, Universität Hamburg, Germany \\ M. LÜSCHER \\ Deutsches Elektronen-Synchrotron DESY, Hamburg, Germany
}

Received 9 July 1979

We compute the determinant of the Dirac operator in a general multi-instanton background field.

\section{Introduction}

Recent studies $[1,2]$ in two-dimensional non-linear $\sigma$-models suggest [3] that the Yang-Mills instanton gas is dense and that the infrared divergencies present in the dilute-gas approximation disappear, when this is properly taken into account. To give this intriguing idea substance, one has to include dense multi-instanton configurations in the semiclassical treatment of the functional integral and is thus led to the problem of computing the determinant of the fluctuation operator in a general multi-instanton background field. We here derive a formula for the determinant of the corresponding Dirac operator, hoping that the determinant of the gluon fluctuation operator can then be computed in a comparatively simple step exploiting supersymmetry [4].

We use the same general method to calculate determinants as in the two-dimensional $\mathrm{CP}^{n-1}$ models [2]. Thus, we first compute the variation $\delta \Gamma$ of

$$
\Gamma=\ln \operatorname{det}^{\prime} \not D, \quad \not D: \text { Dirac operator, }
$$

with respect to the parameters of the instanton background field. This is possible, because

$$
\delta \Gamma=\operatorname{Tr}\{\delta A S\},
$$

$S$ being the known $[5,6]$ Green function of $\not$. Integrating $\delta \Gamma$, one obtains $\Gamma$ up to an integration constant, which is finally computed by considering an especially simple instanton configuration, where all the eigenvalues of $\not D$ can be calculated 
explicitly. Of course, to make $\Gamma$ well-defined, its ultraviolet and infrared divergencies must be regularized. This will be achieved by introducing an appropriate set of Pauli-Villars regulator fields and by projecting the Dirac operator onto a spherical space-time with adjustable world radius $R$.

Since the Green function $S$ is already known, the computation of $\delta \Gamma$ is straightforward, except that the projection onto a spherical space-time causes some calculations to be rather lengthy. The main difficulty, however, is to integrate $\delta \Gamma$, a step which involves a lot of guesswork. For the special case of 't Hooft's instanton solutions [8], this problem simplifies and it has been solved by Brown and Creamer [7].

Our article is organized as follows. To fix notations, the general instanton solution due to Atiyah, Hitchin, Drinfeld and Manin $[9,10]$ for the gauge group $\mathrm{Sp}(r)$ is reviewed in sect. 2. These particular gauge groups are chosen for technical convenience. In fact, no serious limitations arise from this choice, since $\mathrm{Sp}(r)$ contains an $\mathrm{SU}(r)$ subgroup (also, $\mathrm{Sp}(1)=\mathrm{SU}(2)$ ). In sect. 3 we define the Dirac operator on $\mathrm{S}^{4}$, discuss its zero modes and the Green function $S . \delta \Gamma$ is computed subsequently (sect. 4) and integrated in sect. 5. Finally, the integration constant is evaluated in sect. 6 and our results are summarized and commented in the concluding sect. 7 .

\section{Description of the general $\mathrm{Sp}(r)$ instanton solutions}

$\mathrm{Sp}(r)$ can be considered to be the subgroup of $\mathrm{U}(n), n=2 r$, of all those unitary matrices $u$ with *

$$
u^{+}=J u^{\mathrm{T}} J^{\mathrm{T}},
$$

where $J$ is the antisymmetric matrix

$$
J=\left|\begin{array}{rrrrrrrr}
0 & 1 & & & & & & \\
-1 & 0 & & & & 0 & \\
& & & 0 & 1 & & & \\
& & -1 & 0 & & & & \\
& & & & \ddots & & & \\
& 0 & & & & 0 & 1 \\
& & & & & -1 & 0
\end{array}\right| .
$$

Correspondingly, $\mathrm{Sp}(r)$ gauge potentials $A_{\mu}$ are special $\mathrm{U}(n)$ gauge fields, i.e., they are antihermitian $n \times n$ matrices having the additional reality property

$$
A_{\mu}^{+}=J A_{\mu}^{\mathrm{T}} J^{\mathrm{T}}
$$

In particular, $\mathrm{Sp}(r)$ instanton solutions are special cases of $\mathrm{U}(n)$ instanton fields.

\footnotetext{
* T and + denote transposition and hermitian conjugation, respectively. Greek indices $\mu, \nu, \ldots$ run from 0 to 3 , capital Latin indices (spinor indices) $A, B, \ldots A^{\prime}, B^{\prime} \ldots$ from 1 to 2 . The totally antisymmetric tensor $\epsilon_{\mu \nu \rho \sigma}$ is normalized such that $\epsilon_{0123}=+1$ and repeated indices are always summed over.
} 
To explicitly describe these, we need some spinor algebra.

(Multi) spinors are tensors $\xi_{A_{1} \ldots A_{l}, A_{1}^{\prime} \ldots A_{m}^{\prime}}$ with $A_{i}=1,2$ and $A_{j}^{\prime}=1,2$. They carry a representation of the spin covering SU(2) $\times \mathrm{SU}(2)$ of the euclidean Lorentz group $\mathrm{SO}(4)$ :

$$
\begin{aligned}
& {[(u, v) \cdot \xi]_{A_{1} \ldots A_{l}, A_{1}^{\prime} \ldots A_{m}^{\prime}}=u_{A_{1} B_{1}} \ldots u_{A_{l} B} \bar{v}_{A_{1}^{\prime} B_{1}^{\prime}} \ldots \bar{v}_{A_{m}^{\prime} B_{m}^{\prime}} \xi_{B_{1}} \ldots B_{l}, B_{1}^{\prime} \ldots B_{m}^{\prime},} \\
& (u, v) \in \operatorname{SU}(2) \times \operatorname{SU}(2)
\end{aligned}
$$

$\left(\bar{v}_{A^{\prime} B^{\prime}}\right.$ is the complex conjugate of $\left.v_{A^{\prime} B^{\prime}}\right)$. Spinor indices can be raised and lowered, for example

$$
\begin{aligned}
& \xi^{A}=\xi_{B} \epsilon^{B A}, \quad \xi_{A}=\epsilon_{A B} \xi^{B}, \\
& \epsilon_{A B}=\epsilon^{A B}=-\epsilon_{B A}, \quad \epsilon_{12}=1 .
\end{aligned}
$$

Contraction of an upper and a lower index of the same type (i.e., primed or unprimed) is an $\mathrm{SU}(2) \times \mathrm{SU}(2)$ invariant operation. Note that

$$
\xi_{A} \eta^{A}=-\xi^{A} \eta_{A}
$$

The adjoint $\xi_{A}^{+}$of a spinor $\xi_{A}$ is defined by

$$
\xi_{1}^{+}=-\bar{\xi}_{2}, \quad \xi_{2}^{+}=\bar{\xi}_{1}
$$

(analogous formulae are understood for spinors $\eta_{A^{\prime}}$ ). The merit of this construction is that the adjoint spinors transform in the same way under $\operatorname{SU}(2) \times \mathrm{SU}(2)$ as the original spinors. In particular,

$$
(\xi, \eta)=\xi_{A}^{+} \eta^{A}
$$

is an invariant scalar product.

The spinor components $x_{A A^{\prime}}$ of the position vector $x_{\mu}$ are defined by

$$
\begin{aligned}
& x_{A A^{\prime}}=x_{\mu}\left(e_{\mu}\right)_{A A^{\prime}}, \\
& e_{0}=\mathbb{1}, \quad e_{a}=-i \sigma^{a},
\end{aligned}
$$

where $\sigma^{a}, a=1,2,3$, are the Pauli matrices. The covariant matrices $e_{\mu}$ satisfy the following useful identities:

$$
\begin{aligned}
& e_{\mu}^{+}=\epsilon e_{\mu}^{\mathrm{T}} \epsilon^{\mathrm{T}}, \\
& \operatorname{Tr}\left\{e_{\mu}^{+} e_{\nu}\right\}=2 \delta_{\mu \nu}, \quad\left(e_{\mu}\right)_{A A^{\prime}}\left(e_{\mu}\right)_{B B^{\prime}}=2 \epsilon_{A B} \epsilon_{A^{\prime} B^{\prime}} .
\end{aligned}
$$

Now we are well-prepared to describe the $\mathrm{U}(n)$ gauge potentials $A_{\mu}$ giving rise to self-dual field strengths $F_{\mu \nu}[5,6,9,10]$. They can be represented in the form

$$
A_{\mu}=v^{+} \partial_{\mu} v, \quad v^{+} v=1
$$

where $v$ is an $(n+2 k) \times n$ complex matrix. Here, $k$ denotes the instanton number, viz.,

$$
k=-\frac{1}{16 \pi^{2}} \int \mathrm{d}^{4} x \operatorname{Tr}\left(F_{\mu \nu}{ }^{*} F_{\mu \nu}\right), \quad{ }^{*} F_{\mu \nu}=\frac{1}{2} \epsilon_{\mu \nu \rho \sigma} F_{\rho \sigma} .
$$


$v$ is the solution matrix of a set of $2 k$ complex linear equations

$$
v^{+} \Delta_{A^{\prime}}=0, \quad\left(A^{\prime}=1,2\right),
$$

with $\Delta_{A^{\prime}}$ a spinor of $(n+2 k) \times k$ matrices depending linearly on $x_{\mu}$ :

$$
\Delta_{A^{\prime}}=a_{A^{\prime}}+b^{A} x_{A A^{\prime}} .
$$

$a_{A^{\prime}}$ and $b_{A}$ are constant $(n+2 k) \times k$ matrices, which parametrize the instanton solution. They are not unconstrained, however, but must be chosen such that

$$
\Delta_{A^{\prime}}^{+} \Delta_{B^{\prime}}=-\epsilon_{A^{\prime} B^{\prime}} f^{-1}, \quad \text { for all } x \text {. }
$$

Here, $\Delta_{A^{\prime}}^{+}$is the adjoint of $\Delta_{A^{\prime}}$ in the sense of spinors (cf., eq. (6)), viz.,

$$
\Delta_{1}^{+}=-\bar{\Delta}_{2}^{\mathbf{T}}, \quad \Delta_{2}^{+}=\bar{\Delta}_{1}^{\mathrm{T}} .
$$

Noting

$$
\Delta_{A^{\prime}}^{+}=a_{A^{\prime}}^{+}+b^{+A} x_{A A^{\prime}},
$$

it follows that eq. (15) holds if and only if

$$
\begin{aligned}
& a_{A^{\prime}}^{+} a_{B^{\prime}}+a_{B^{\prime}}^{+} a_{A^{\prime}}=0, \\
& a_{A}^{+} b_{A}+b_{A}^{+} a_{A^{\prime}}=0, \\
& b_{A}^{+} b_{B}+b_{B}^{+} b_{A}=0 .
\end{aligned}
$$

Thus, given a pair of parameter matrices $a_{A^{\prime}}, b_{A}$ satisfying these quadratic constraints, the gauge potential $A_{\mu}$ constructed via eqs. (13) and (11) has self-dual curvature. It will be non-singular provided the $k \times k$ matrix $f^{-1}$ defined by eq. (15) is invertible for all $x$.

Two sets $\left(a_{A^{\prime}}, b_{A}\right)$ and $\left(\hat{a}_{A^{\prime}}, \hat{b}_{A}\right)$ of parameter matrices related by

$$
a_{A^{\prime}}=U \hat{a}_{A^{\prime}} K, \quad b_{A}=U \hat{b}_{A} K, \quad(U \in \mathrm{U}(n+2 k) ; K \in \mathrm{Gl}(k, \mathbb{C}))
$$

give rise to the same instanton solution. This invariance can be exploited to transform $b_{A}$ to its "normal form":

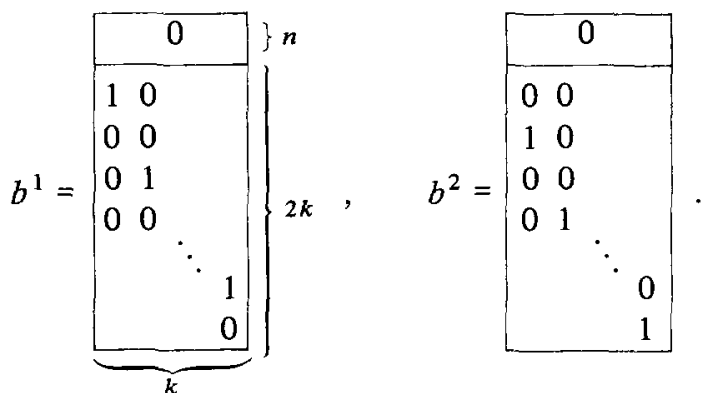

We shall henceforth assume that $b_{A}$ is in its normal form. 
$\mathrm{U}(n)$ instanton potentials $A_{\mu}$, which are reducible to $\mathrm{Sp}(r)$, come from $a_{A}$ 's having the reality property (cf., eq. (2))

$$
a_{A^{\prime}}^{+}=a_{A^{\prime}}^{\mathrm{T}} J^{\mathrm{T}} \text {. }
$$

The analogous equation for $b_{A}$,

$$
b_{A}^{+}=b_{A}^{\mathrm{T}} J^{\mathrm{T}},
$$

holds automatically, when $b_{A}$ is in the normal form (19). Eqs. (20) imply in particular that $f$ is a real symmetric $k \times k$ matrix for $\mathrm{Sp}(r)$ instantons.

\section{The Dirac operator on $\mathbf{S}^{4}$ and its Green function}

Analogous to the $\mathrm{CP}^{n-1}$ case [2], one could set up a manifestly $\mathrm{O}(5)$ covariant formalism to deal with Dirac fields and gauge potentials on $\mathrm{S}^{4}$. We here prefer, however, to work with explicit stereographic coordinates $x_{\mu}$ instead. Thus, points $r_{\alpha}$, $\alpha=0, \ldots, 4, r_{\alpha} r_{\alpha}=R^{2}$, of $S^{4}$ are parametrized by $x_{\mu}$ according to

$$
r_{\mu}=\frac{2 R^{2} x_{\mu}}{R^{2}+x^{2}}, \quad r_{4}=R \frac{R^{2}-x^{2}}{R^{2}+x^{2}} .
$$

In these coordinates, the natural metric on $\mathrm{S}^{4}$ is

$$
g_{\mu \nu}=\Omega \delta_{\mu \nu}, \quad \Omega=4 R^{4}\left(R^{2}+x^{2}\right)^{-2},
$$

and the Dirac operator reads *

$$
D=2 \Omega^{-1 / 2} i \gamma_{\mu} D_{\mu}, \quad D_{\mu}=\partial_{\mu}+A_{\mu}
$$

The $\gamma$-matrices are defined by

$$
\gamma_{\mu}=\left(\begin{array}{ll}
0 & e_{\mu} \\
e_{\mu}^{+} & 0
\end{array}\right)
$$

The gauge potential $A_{\mu}$ is taken to be any $\operatorname{Sp}(r) k$-instanton solution as described above. In particular, the Dirac fields, on which $D$ acts, are in the quark representation of the color group. The factor of 2 in eq. (23) is added to insure that $D$ formally approaches the usual flat-space Dirac operator in the infinite volume limit $R \rightarrow \infty$.

\footnotetext{
\Many authors prefer a normalization of Dirac fields different from ours, corresponding to a Dirac operator

$$
\hat{D}=\Omega^{-3 / 4} D \Omega^{3 / 4}=2 \Omega^{-5 / 4} i \gamma_{\mu} D \Omega^{3 / 4}
$$

Both formulations are completely equivalent, of course.
} 
A characteristic property of the Dirac operator is its off-diagonal shape

$$
D=\left(\begin{array}{ll}
0 & T^{+} \\
T & 0
\end{array}\right),
$$

where

$$
T=2 \Omega^{-1 / 2} i e_{\mu}^{+} D_{\mu}, \quad T^{+}=2 \Omega^{-1 / 2} i e_{\mu} D_{\mu} .
$$

Writing $\left(e_{\mu}^{+}\right)^{A^{\prime} A}$ for the matrix elements of $e_{\mu}^{+}$, we see that $T$ maps spinors $\psi_{A}$ of negative chirality onto spinors $\chi^{A^{\prime}}$ of positive chirality. These two spaces $V_{\ldots}$ and $V_{+}$are equipped with the natural $O(5)$ invariant scalar products

$$
\begin{aligned}
& (\psi, \varphi)=\int \mathrm{d}^{4} x \Omega^{1 / 2} \psi_{A}^{+} \varphi^{A}, \\
& (\chi, \xi)=\int \mathrm{d}^{4} x \Omega^{1 / 2} \chi_{A^{\prime}}^{+} \xi^{A^{\prime}}
\end{aligned}
$$

(contraction of color indices is understood). Correspondingly, $T^{+}$is the adjoint of $T$ mapping $\mathrm{V}_{+}$into $V_{-}$.

$T^{+}$has no zero modes, but $T$ has exactly $k$, viz. $[5,11]$,

$$
\psi_{\alpha, A}^{i}=\left(v^{+} b_{A} f\right)_{\alpha i}
$$

Here $i=1, \ldots, k$ labels the solutions, $\alpha=1, \ldots, n$ is the color index and $A=1,2$ the spinor index. The projector $P_{0}$ onto the zero modes (27) can be written as an integral operator

$$
\left(P_{0} \psi\right)_{A}(x)=\int \mathrm{d}^{4} y \Omega(y)^{1 / 2} P_{0}(x, y)_{A B} \psi^{B}(y),
$$

where

$$
\begin{aligned}
& P_{0}(x, y)_{A B}=\sum_{i, j} \psi_{A}^{i}(x) N_{i j}^{-1} \psi_{B}^{j}(y)^{+}, \\
& N_{i j}=\left(\psi^{i}, \psi^{j}\right) .
\end{aligned}
$$

Color indices are to be contracted appropriately, of course, and adjoints are taken in the spinor sense, eq. (6).

The Green function $S$ of the Dirac operator $D$ is off-diagonal as well:

$$
S=\left(\begin{array}{ll}
0 & S_{-} \\
S_{+} & 0
\end{array}\right) .
$$

Thus, $S_{-}$maps $\mathrm{V}_{+}$into $\mathrm{V}_{-}$and $S_{+}$is the adjoint of $S_{-}$. The defining equations for $S_{-}$are

$$
T S_{-}=1, \quad P_{0} S_{-}=0 .
$$

More explicitly, defining an integral kernel $S_{-}(x, y)_{A A^{\prime}}$ by

$$
\left(S_{-} \psi\right)_{A}(x)=\int \mathrm{d}^{4} y \Omega(y)^{1 / 2} S_{-}(x, y)_{A A^{\prime}} \psi^{A^{\prime}}(y),
$$


eq. (31) reads

$$
\begin{aligned}
& T^{A^{\prime} A} S_{-}(x, y)_{A B^{\prime}}=\Omega^{-1 / 2} \delta_{B^{\prime}}^{A^{\prime}} \delta(x-y), \\
& \int \mathrm{d}^{4} y \Omega(y)^{1 / 2} P_{0}(x, y)_{A B} S_{-}(y, z)^{B}{ }_{B^{\prime}}=0 .
\end{aligned}
$$

The solution of these equations is not difficult to guess:

$$
\begin{aligned}
& S_{-}(x, y)_{A A^{\prime}}=\hat{S}_{-}(x, y)_{A A^{\prime}}-\int \mathrm{d}^{4} z \Omega(z)^{1 / 2} P_{0}(x, z)_{A B} \hat{S}_{-}(z, y)^{B} A^{\prime}, \\
& \hat{S}_{-}(x, y)_{A A^{\prime}}=\frac{1}{2} i\left(e_{\mu}\right)_{A A^{\prime}} D_{\mu} G(x, y) .
\end{aligned}
$$

Here, $G(x, y)$ denotes the known scalar Green function $[5,6]$

$$
G(x, y)=\frac{v^{+}(x) \cdot v(y)}{4 \pi^{2}(x-y)^{2}}, \quad D_{\mu} D_{\mu} G(x-y)=-\delta(x-y) .
$$

The simple solution (34) can be traced back to the simple conformal transformation properties of the Dirac operator. In particular, we fear that it would be much harder to find the Green function for the gluon fluctuation operator on $\mathrm{S}^{4}$.

\section{Computation of the variation of the determinant of the Dirac operator}

Because $\gamma_{5}$ anticommutes with $D$, its non-zero eigenvalues come in pairs $(\lambda,-\lambda)$. The overall sign of det $D$ is therefore ambiguous. We remove this ambiguity by defining

$$
\Gamma=\ln \operatorname{det}^{\prime} D \stackrel{\operatorname{def}}{=} \frac{1}{2} \ln \operatorname{det}^{\prime} D^{2} .
$$

The prime indicates that the zero modes of $D$ should be omitted. To regularize the UV divergence of $\Gamma$ we now add Pauli-Villars regulator fields with large masses $M_{i}$ and alternating "metric" $e_{i}$, such that

$$
\sum_{i=1}^{\nu} e_{i}=-1, \quad \sum_{i=1}^{\nu} e_{i} M_{i}^{2 p}=0, \quad(p=1, \ldots, \nu-1) .
$$

When the number $\nu$ of regulator fields is greater than 3 , the regularized determinant

$$
\Gamma_{\text {reg }}=\frac{1}{2} \operatorname{Tr}\left\{\ln \left(D^{2}+P_{0}\right)+\sum_{i=1}^{\nu} e_{i} \ln \left(D^{2}+M_{i}^{2}\right)\right\}
$$

is finite and perfectly well-defined. Eq. (38) can be given a somewhat simpler form noting that

$$
D^{2}=\left(\begin{array}{ll}
T^{+} T & 0 \\
0 & T T^{+}
\end{array}\right)
$$


and that the non-zero eigenvalues of $T^{+} T$ and $T T^{+}$are identical:

$$
\Gamma_{\mathrm{reg}}=k \sum_{i=1}^{\nu} e_{i} \ln M_{i}+\operatorname{Tr}\left\{\ln \left(T T^{+}\right)+\sum_{i=1}^{\nu} e_{i} \ln \left(T T^{+}+M_{i}^{2}\right)\right\} .
$$

The trace here is to be taken in the space $V_{+}$of normalizable spinor fields $\chi_{A}{ }^{\prime}$.

We now consider a curve $A_{\mu}(x, s), 0 \leqslant s \leqslant 1$, of instanton solutions with $A_{\mu}(x, 0)=A_{\mu}(x)$. Our aim is to compute

$$
\delta \Gamma_{\text {reg }}=\left.\frac{\mathrm{d}}{\mathrm{d} s} \Gamma_{\text {reg }}\right|_{s=0} .
$$

From eq. (39) it follows that

$$
\delta \Gamma_{\text {reg }}=\operatorname{Tr}\left\{\delta T\left[T^{+}\left(T T^{+}\right)^{-1}+\sum_{i=1}^{\nu} e_{i} T^{+}\left(T T^{+}+M_{i}^{2}\right)^{-1}\right\}\right\}+\text { c.c. }
$$

where c.c. means complex conjugate. Noting $T^{+}\left(T T^{+}\right)^{-1}=S_{-}$and defining $S_{-}^{M}(x, y)_{A A^{\prime}}$ to be the integral kernel of $T^{+}\left(T T^{+}+M^{2}\right)^{-1}$, this becomes

$$
\begin{aligned}
& \delta \Gamma_{\text {reg }}=\int \mathrm{d}^{4} y \operatorname{Tr}_{\text {color }}\left\{2 i\left(e_{\mu}^{+}\right)^{A^{\prime} A} \delta A_{\mu}(y)\right. \\
& \left.\quad \times\left[S_{-}(x, y)_{A A^{\prime}}+\sum_{i=1}^{\nu} e_{i} S_{-}^{M_{i}}(x, y)_{A A^{\prime}}\right]_{x=y}\right\}+ \text { c.c. }
\end{aligned}
$$

Thus, to compute $\delta \Gamma_{\text {reg }}$ we must study the short-distance behaviour of $S_{-}$and $S_{-}^{M}$. From the explicit form, eqs. (34), (35), of $S_{-}$one derives that up to terms vanishing at $x=y$

$$
\begin{aligned}
& S_{-}(x, y)_{A A^{\prime}}=\frac{1}{2} i\left(e_{\mu}\right)_{A A^{\prime}} D_{\mu}\left[U(x, y)\left(4 \pi^{2}(x-y)^{2}\right)^{-1}\right] \\
& \quad-\frac{1}{24 \pi^{2}}\left[3\left(D_{\mu} v^{+}\right) b^{B} f b_{B}^{+} v+v^{+} b^{B} f b_{B}^{+}\left(D_{\mu} v\right)+v^{+} b^{B} \partial_{\mu} f b_{B}^{+} v\right](y) \\
& \quad-\int \mathrm{d}^{4} z \Omega(z)^{1 / 2} P_{0}(y, z)_{A B} \hat{S}_{-}(z, y)^{B} A^{\prime} .
\end{aligned}
$$

Here, $U(x, y)$ is the gauge parallel transporter along the straight line from $y$ to $x$ :

$$
U(x, y)=\mathrm{P} \exp -\int_{y}^{x} \mathrm{~d} z_{\mu} A_{\mu}(z), \quad \mathrm{P}: \text { path ordering. }
$$

On the other hand, the short-distance behaviour of $S_{-}^{M}$ can be calculated perturbatively (see appendix):

$$
\sum_{i=1}^{\nu} e_{i} S_{-}^{M_{i}}(x, y)_{A A^{\prime}}=-\frac{1}{2} i\left(e_{\mu}\right)_{A A^{\prime}}\left\{D_{\mu}\left[U(x, y)\left(4 \pi^{2}(x-y)^{2}\right)^{-1}\right]\right.
$$




$$
\left.-F_{\mu \nu}(y) y_{\nu} \frac{\Omega(y)^{1 / 2}}{24 \pi^{2} R^{2}}\right\} \text {. }
$$

Terms which vanish in the limit where first $x \rightarrow y$ and then $M_{i} \rightarrow \infty$ have been neglected here.

When inserting eqs. (41) and (43) into eq. (40) we see that the short-distance singularities cancel and we are left with

$$
\begin{aligned}
& \delta \Gamma_{\text {reg }}=\delta \Gamma_{1}+\delta \Gamma_{2}+\frac{1}{6 \pi^{2}} \int \mathrm{d}^{4} x \operatorname{Tr}\left\{\delta A_{\mu}(x) j_{\mu}(x)\right\} \\
& \delta \Gamma_{1}=-\int \mathrm{d}^{4} y \mathrm{~d}^{4} z \operatorname{Tr}\left\{2 i\left(e_{\mu}^{+}\right)^{A^{\prime} A}\right. \\
& \left.\quad \times \delta A_{\mu}(y) P_{0}(y, z)_{A B} \Omega(z)^{1 / 2} \hat{S}_{-}(z, y)^{B} A^{\prime}\right\}+ \text { c.c. }, \\
& \delta \Gamma_{2}=-\left(6 \pi^{2} R^{2}\right)^{-1} \int \mathrm{d}^{4} y \Omega(y)^{1 / 2} y_{\nu} \operatorname{Tr}\left\{\delta A_{\mu}(y) F_{\mu \nu}(y)\right\} \\
& j_{\mu}=v^{+}\left\{b^{A} f b_{A}^{+} \Delta^{A^{\prime}} f \partial_{\mu} \Delta_{A^{\prime}}^{+}-\partial_{\mu} \Delta^{A^{\prime}} f \Delta_{A^{\prime}}^{+} b^{A} f b_{A}^{+}\right\} v .
\end{aligned}
$$

$\delta \Gamma_{1}$ can be simplified as follows. Denote by $\hat{T}$ and $\hat{P}_{0}$ the operators $T$ and $P_{0}$ with $\Omega$ set equal to one. Then

$$
\delta \Gamma_{1}=-\operatorname{Tr}\left\{\delta \hat{T} P_{0} \Omega^{1 / 2} \hat{T}^{+}\left(\hat{T} \hat{T}^{+}\right)^{-1}\right\}+\text { c.c. }
$$

Now $\hat{T} P_{0}=0$ so that

$$
\delta \hat{T} P_{0}=-\hat{T} \delta P_{0} .
$$

Furthermore,

$$
\hat{T}^{+}\left(\hat{T} \hat{T}^{+}\right)^{-1} \hat{T}=1-\hat{P}_{0},
$$

and therefore

$$
\begin{aligned}
\delta \Gamma_{1} & =\operatorname{Tr}\left\{\delta P_{0} \Omega^{1 / 2}\left(1-\hat{P}_{0}\right)\right\}+\text { c.c. } \\
& =\delta \operatorname{Tr}(\ln N-\ln \hat{N})
\end{aligned}
$$

( $N$ is the zero-mode matrix (29)).

$\delta \Gamma_{2}$ can also be integrated easily. Namely, using the field equation $D_{\mu} F_{\mu \nu}=0$, we have

$$
\delta \operatorname{Tr}\left\{F_{\mu \nu} F_{\mu \nu}\right\}=4 \partial_{\mu} \operatorname{Tr}\left\{\delta A_{\nu} F_{\mu \nu}\right\},
$$

so that

$$
\begin{aligned}
\delta \Gamma_{2} & =-\frac{1}{12 \pi^{2}} \int \mathrm{d}^{4} x\left(\partial_{\mu} \ln \Omega\right) \operatorname{Tr}\left\{\delta A_{\nu} F_{\mu \nu}\right\} \\
& =\delta\left[\frac{1}{48 \pi^{2}} \int \mathrm{d}^{4} x \ln \Omega \operatorname{Tr}\left\{F_{\mu \nu} F_{\mu \nu}\right\}\right] .
\end{aligned}
$$


The rest of $\delta \Gamma$ will be integrated in sect. 5. As a preliminary step towards this goal, we here derive an expression for $\delta A_{\mu}$ in terms of $\delta a_{A^{\prime}}$, the variation of the instanton parameter matrix ( $\delta b_{A}=0$ since $b_{A}$ is always assumed to be in its normal form (19)). Defining the projector

$$
P=v v^{+}=1-\Delta^{A^{\prime}} f \Delta_{A^{\prime}}^{+},
$$

it is easy to check that

$$
\delta A_{\mu}=v^{+}\left[\delta P, \partial_{\mu} P\right] v+D_{\mu}\left(v^{+} \delta v\right) .
$$

The term $D_{\mu}\left(v^{+} \delta v\right)$ can be dropped, because $\delta \Gamma_{\text {reg }}$ is gauge invariant (or, equivalently, since $D_{\mu} j_{\mu}=0$ ). Inserting eq. (44) into eq. (45) gives $\delta A_{\mu}$ as a function of $\delta a_{A^{\prime}}$.

For ease of reference, we finally collect the results of this section to obtain

$$
\begin{aligned}
& \delta \Gamma_{\text {reg }}=\delta\left\{\operatorname{Tr}(\ln N-\ln \hat{N})+\frac{1}{48 \pi^{2}} \int \mathrm{d}^{4} x \ln \Omega \operatorname{Tr}\left(F_{\mu \nu} F_{\mu \nu}\right)\right\} \\
& +\frac{1}{6 \pi^{2}} \int \mathrm{d}^{4} x \operatorname{Tr}\left(\delta A_{\mu} j_{\mu}\right) .
\end{aligned}
$$

Here, the $k \times k$ matrices $N$ and $\hat{N}$ are given by ( $c f$., eq. (27))

$$
\begin{aligned}
& N_{i j}=\int \mathrm{d}^{4} x \Omega^{1 / 2} \psi_{A}^{i+} \psi^{j A}, \\
& \hat{N}_{i j}=\int \mathrm{d}^{4} x \psi_{A}^{i+} \psi^{j A},
\end{aligned}
$$

and, with $d_{A^{\prime}} \stackrel{\text { def }}{=} \delta a_{A^{\prime}}$,

$$
\begin{aligned}
& \delta A_{\mu}=v^{+}\left\{d^{A^{\prime}} f \partial_{\mu} \Delta_{A^{\prime}}^{+}-\partial_{\mu} \Delta^{A^{\prime}} f d_{A^{\prime}}^{+}\right\} v, \\
& j_{\mu}=v^{+}\left\{b^{A} f b_{A}^{+} \Delta^{A^{\prime}} f \partial_{\mu} \Delta_{A^{\prime}}^{+}-\partial_{\mu} \Delta^{A^{\prime}} f \Delta_{A^{\prime}}^{+} b^{A} f b_{A}^{+}\right\} v .
\end{aligned}
$$

\section{Integration of $\delta \Gamma_{\text {reg }}$}

Of course, $\Gamma_{\text {reg }}$ can be obtained by integrating $\delta \Gamma_{\text {reg }}$ along any path of instanton solutions connecting $A_{\mu}$ with some standard configuration $A_{\mu}^{0}$, thus giving $\Gamma_{\text {reg }}$ essentially as a five-dimensional integral. Such an expression is quite implicit, however, because it is difficult to display the integration paths. In this section we derive a formula for $\Gamma_{\text {reg }}$ involving a five-dimensional integral too, but with an integrand which is an explicit rational function of the instanton parameters and the integration variables.

We first rewrite $\operatorname{Tr}\left(\delta A_{\mu} j_{\mu}\right)$ in terms of the basic matrices $b_{A}, \Delta_{A^{\prime}}$ and $f$. From 
eqs. (10), (44) and (48) one finds

$$
\begin{gathered}
\operatorname{Tr}\left(\delta A_{\mu} j_{\mu}\right)=2 \operatorname{Tr}\left\{\underline{d f b^{+} P \overrightarrow{b f b}} \bar{u}^{+} \Delta f b^{+} P\right. \\
\left.+d f b^{+} P b f \Delta^{+} \widehat{b f b}^{+} P\right\}+ \text { c.c. }
\end{gathered}
$$

The lines connecting pairs of matrices imply that their spinor indices are contracted such that the index of the left matrix is in the upper position, e.g.,

$$
\overrightarrow{b f b}^{+}=b^{A} f b_{A}^{+} \text {. }
$$

By adding a suitable divergence $\partial_{\mu} \Lambda_{\mu}$ one can express $\operatorname{Tr}\left(\delta A_{\mu} j_{\mu}\right)$ through $f$ alone:

$$
\begin{aligned}
& \operatorname{Tr}\left(\delta A_{\mu} j_{\mu}\right)+\partial_{\mu} \Lambda_{\mu} \\
& \quad=\frac{1}{4} T_{1}-\frac{3}{4} T_{2}+\frac{3}{4} T_{3}+\frac{1}{4} T_{4}+T_{5}-\frac{1}{2} T_{6}-T_{7}+T_{8}-3 T_{9} .
\end{aligned}
$$

Here,

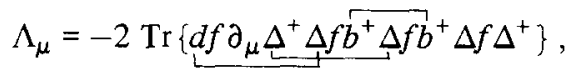

and the $T_{i}$ 's are the following traces:

$$
\begin{aligned}
& T_{1}=\epsilon_{\mu \nu \rho \sigma} \operatorname{Tr}\left\{f \delta f^{-1} f \partial_{\mu} f^{-1} f \partial_{\nu} f^{-1} f \partial_{\rho} f^{-1} f \partial_{\sigma} f^{-1}\right\}, \\
& T_{2}=\operatorname{Tr}\left\{f \delta f^{-1} f \partial_{\mu} f^{-1} f \partial_{\mu} f^{-1} f \partial_{\nu} f^{-1} f \partial_{\nu} f^{-1}\right\}, \\
& T_{3}=\operatorname{Tr}\left\{f \delta f^{-1} f \partial_{\mu} f^{-1} f \partial_{\nu} f^{-1} f \partial_{\mu} f^{-1} f \partial_{\nu} f^{-1}\right\}, \\
& T_{4}=\operatorname{Tr}\left\{f \delta f^{-1} f \partial_{\mu} f^{-1} f \partial_{\nu} f^{-1} f \partial_{\nu} f^{-1} f \partial_{\mu} f^{-1}\right\}, \\
& T_{5}=\operatorname{Tr}\left\{f \partial_{\mu} \delta f^{-1} f \partial_{\mu} f^{-1} f \partial_{\nu} f^{-1} f \partial_{\nu} f^{-1}\right\}, \\
& T_{6}=\operatorname{Tr}\left\{f \partial_{\mu} \delta f^{-1} f \partial_{\nu} f^{-1} f \partial_{\mu} f^{-1} f \partial_{\nu} f^{-1}\right\}, \\
& T_{7}=\operatorname{Tr}\left\{f \delta f^{-1} f \bar{b} b f \partial_{\mu} f^{-1} f \partial_{\mu} f^{-1}\right\}, \\
& T_{8}=\operatorname{Tr}\left\{f \delta f^{-1} f \partial_{\mu} f^{-1} f b^{+} b f \partial_{\mu} f^{-1}\right\}, \\
& T_{9}=\operatorname{Tr}\left\{f \partial_{\mu} \delta f^{-1} f \partial_{\mu} f^{-1} f b^{+} b\right\}, \\
& T_{10}=\operatorname{Tr}\left\{f \delta f^{-1} f \sqrt{b^{+} b} f b^{+} b\right\}
\end{aligned}
$$

( $T_{10}$ is added to the list for later convenience). We only know of a rather lengthy proof of eq. (50). It goes as follows. First observe that using the rules

$$
\begin{aligned}
& \delta f^{-1}=\frac{1}{2}\left(d_{A^{\prime}}^{+} \Delta^{A^{\prime}}+\Delta_{A^{\prime}}^{+} d^{A^{\prime}}\right),
\end{aligned}
$$

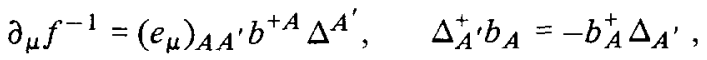

all $T_{i}$ 's can be written as linear combinations of traces of products of

$$
\begin{aligned}
& d_{A^{\prime}} f b_{A}^{+}, \quad b_{A} f d_{A^{\prime}}^{+}, \quad \Delta_{A^{\prime}} f d_{B^{\prime}}^{+} ; \\
& \Delta_{A}^{\prime} f \Delta_{B^{\prime}}^{+}, \quad \Delta_{A} f b_{A}^{+}, \quad b_{A} f \Delta_{A^{\prime}}^{+}, \quad b_{A} f b_{B}^{+},
\end{aligned}
$$


with all spinor indices contracted. All terms contain precisely one matrix $d_{A^{\prime}}$ or $d_{A^{\prime}}^{+}$ and four matrices $b_{A}$ or $b_{A}^{+}$. Such traces are called basic. For example, the expansion of $T_{2}$ in basic traces is

$$
\begin{aligned}
T_{2} & =\frac{1}{2} \operatorname{Tr}\{\underbrace{d f b^{+} \Delta f b^{+} \Delta f b^{+} \Delta f b^{+} \Delta f \Delta^{+}}\} \\
& +\frac{1}{2} \operatorname{Tr}\{\underbrace{\Delta f d^{+} \Delta f b^{+} \Delta f b^{+} \Delta f b^{+} \Delta f b^{+}}\} .
\end{aligned}
$$

It is obvious from eqs. (49) and (51) that the left-hand side of eq. (50) can also be written as a linear combination of basic traces. The two sides of eq. (50) do not yet match, however, because the basic traces are not linearly independent.

There are three sources for linear relations between basic traces. First, the reality property eq. (20) of $\mathrm{Sp}(r)$ instanton parameter matrices implies that all basic traces are real. Any trace involving $d_{A}^{+}$is therefore equal to another basic trace containing $d_{A^{\prime}, \text { e.g., }}$

$$
\operatorname{Tr}\{\underbrace{\Delta f d^{+} \Delta f b^{+} \Delta f b^{+}} \Delta f b^{+} \Delta f b^{+}\}=\operatorname{Tr}\{\underbrace{d f b^{+} \Delta f b^{+} \Delta f b^{+} \Delta f b^{+} \Delta f \Delta^{+}}\} .
$$

A second set of relations between basic traces arises from the rule

$$
\epsilon_{A^{\prime} B^{\prime}} \epsilon_{C^{\prime} D^{\prime}}=\epsilon_{A^{\prime} C^{\prime}} \epsilon_{B^{\prime} D^{\prime}}-\epsilon_{A^{\prime} D^{\prime}} \epsilon_{B^{\prime} C^{\prime}}
$$

which reads graphically

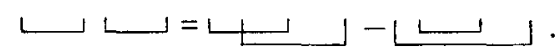

Of course, the analogous rule holds for contractions of unprimed indices, too. Note also that

$$
b_{A}^{+} b_{B}=\frac{1}{2} \epsilon_{A B} \sqrt{b^{+} b} .
$$

A third type of relations is finally obtained from

$$
\begin{aligned}
& d_{A^{\prime}}^{+} \Delta_{B^{\prime}}+\Delta_{A^{\prime}}^{+} d_{B^{\prime}}+d_{B^{\prime}}^{+} \Delta_{A^{\prime}}+\Delta_{B^{\prime}}^{+} d_{A^{\prime}}=0, \\
& d_{A^{\prime} b_{A}}+b_{A}^{+} d_{A^{\prime}}=0
\end{aligned}
$$

(this is a consequence of eq. (15), (18b), respectively). For example, using reality, we have

$$
\begin{aligned}
B_{1} & \stackrel{\text { def }}{=} \operatorname{Tr}\{\underbrace{\Delta^{+} d f b^{+} \Delta f b^{+} \Delta f b^{+} \Delta f b^{+}} \Delta f\} \\
& =\operatorname{Tr}\{\underbrace{d^{+} \Delta f b^{+} \Delta f b^{+} \Delta f b^{+} \Delta f b^{+}} \Delta f\}, \\
B_{2} & \stackrel{\text { def }}{=} \operatorname{Tr}\{\underbrace{\Delta^{+} d f b^{+} \Delta f b^{+} \Delta f b^{+} \Delta f b^{+}} \Delta f\} \\
& =\operatorname{Tr}\{\underbrace{d^{+} \Delta f b^{+} \Delta f b^{+} \Delta f b^{+} \Delta f b^{+} \Delta f}\},
\end{aligned}
$$

and therefore $B_{1}+B_{2}=0$. 
As a result of all these linear dependences, any basic trace can be represented as a linear combination of the elements of an irreducible set of 14 basic traces. When this is done for both sides of eq. (50), they turn out to be identical.

Most of the right-hand side of eq. (50) can be integrated quite easily, viz.,

$$
\begin{aligned}
-\frac{3}{4} & T_{2}+\frac{3}{4} T_{3}+\frac{1}{4} T_{4}+T_{5}-\frac{1}{2} T_{6}-T_{7}+T_{8}-3 T_{9} \\
& =\delta\left(\frac{1}{4} D_{1}-5 D_{2}\right)+\partial_{\mu}\left(\frac{1}{8} \Lambda_{\mu}^{1}-\frac{7}{16} \Lambda_{\mu}^{2}+\frac{1}{16} \Lambda_{\mu}^{3}-\frac{5}{4} \Lambda_{\mu}^{4}-\frac{7}{2} \Lambda_{\mu}^{5}\right) \\
D_{1} & =\operatorname{Tr}\left\{f \partial_{\mu} f^{-1} f \partial_{\mu} f^{-1} f \partial_{\nu} f^{-1} f \partial_{\nu} f^{-1}\right\}, \\
D_{2} & =\frac{1}{4} \operatorname{Tr}\left\{\check{b}^{+} b f b^{+} b\right\} \\
\Lambda_{\mu}^{1} & =\operatorname{Tr}\left\{f \delta f^{-1} f \partial_{\mu} f^{-1} f \partial_{\nu} f^{-1} f \partial_{\nu} f^{-1}\right\}, \\
\Lambda_{\mu}^{2} & =\operatorname{Tr}\left\{f \delta f^{-1} f \partial_{\nu} f^{-1} f \partial_{\mu} f^{-1} f \partial_{\nu} f^{-1}\right\}, \\
\Lambda_{\mu}^{3} & =\operatorname{Tr}\left\{f \partial_{\mu} \delta f^{-1} f \partial_{\nu} f^{-1} f \partial_{\nu} f^{-1}\right\}, \\
\Lambda_{\mu}^{4} & =-\frac{1}{2} \operatorname{Tr}\left\{f \delta f^{-1} f b^{+} b f \partial_{\mu} f^{-1}\right\} \\
\Lambda_{\mu}^{5} & =-\frac{1}{2} \operatorname{Tr}\left\{f \partial_{\mu} \delta f^{-1} f b^{+} b\right\} .
\end{aligned}
$$

Eq. (52) is proved straightforwardly by computing $\delta D_{i}$ and $\partial_{\mu} \Lambda_{\mu}^{i}$, observing the rules

$$
\delta f=-f \delta f^{-1} f, \quad \partial_{\mu} f=-f \partial_{\mu} f^{-1} f, \quad \partial_{\mu} \partial_{\nu} f^{-1}=2 \delta_{\mu \nu},
$$

and expressing the result as linear combinations of $T_{2} \ldots T_{10}$ (as usual, we assume that $b_{A}$ is in its normal form, in particular that $\overline{b^{+} b}=-2$ ). For example,

$$
\begin{aligned}
& \delta D_{1}=-2 T_{2}-2 T_{4}+4 T_{5}, \\
& \partial_{\mu} \Lambda_{\mu}^{4}=T_{7}+\frac{1}{2} T_{8}-\frac{1}{2} T_{9}+2 T_{10} .
\end{aligned}
$$

Summarizing eqs. (50) and (52) we obtain

$$
\int \mathrm{d}^{4} x \operatorname{Tr}\left(\delta A_{\mu} j_{\mu}\right)=\int \mathrm{d}^{4} x\left\{\frac{1}{4} T_{1}+\frac{1}{4} \delta D_{1}-5 \delta D_{2}\right\}
$$

The final step, the integration of $T_{1}$, requires a little detour. Let $M(\xi) \in \mathrm{G} 1(k, \mathbb{C})$ be an arbitrary function of five real variables $\xi_{0}, \ldots, \xi_{4}$. Define

$$
q(\xi)=\epsilon_{\alpha \beta \gamma \delta \lambda} \operatorname{Tr}\left\{M^{-1} \partial_{\alpha} M M^{-1} \partial_{\beta} M M^{-1} \partial_{\gamma} M M^{-1} \partial_{\delta} M M^{-1} \partial_{\lambda} M\right\} .
$$

$q(\xi)$ has a topological character in the sense that if $M$ is varied, $q$ changes by a local divergence only:

$$
\delta q=5 \partial_{\alpha} \epsilon_{\alpha \beta \gamma \delta \lambda} \operatorname{Tr}\left\{M^{-1} \delta M M^{-1} \partial_{\beta} M M^{-1} \partial_{\gamma} M M^{-1} \partial_{\delta} M M^{-1} \partial_{\lambda} M\right\} .
$$

An integrated form of this equation is

$$
q=5 \partial_{\alpha} \int_{0}^{1} \mathrm{~d} t \epsilon_{\alpha \beta \gamma \delta \lambda} \operatorname{Tr}\left\{K^{-1} \partial_{t} K K^{-1} \partial_{\beta} K \ldots K^{-1} \partial_{\lambda} K\right\}
$$


where $K(t, \xi), 0 \leqslant t \leqslant 1$, is any curve of invertible matrices such that $K(0, \xi)$ is diagonal and $K(1, \xi)=M(\xi)$.

To apply eq. (56) to the problem at hand, we identify $\xi_{\mu}$ with $x_{\mu}(\mu=0, \ldots, 3)$, $\xi_{4}$ with any instanton parameter such that $\delta=\partial_{4}$, and $M$ with $f^{-1}$. Then,

$$
T_{1}=\epsilon_{\mu \nu \rho \sigma} \operatorname{Tr}\left\{f \partial_{4} f^{-1} f \partial_{\mu} f^{-1} f \partial_{\nu} f^{-1} f \partial_{\rho} f^{-1} f \partial_{\sigma} f^{-1}\right\}=\frac{1}{5} q .
$$

Furthermore, choosing

$$
K_{i j}=(1-t)\left(1+x^{2}\right) \delta_{i j}+t f_{i j}^{-1},
$$

which is strictly positive and therefore invertible for all $t$ and $x$, eq. (56) becomes

$$
T_{1}=\delta \int_{0}^{1} \mathrm{~d} t \epsilon_{\mu \nu \rho \sigma} \operatorname{Tr}\left\{K^{-1} \partial_{t} K K^{-1} \partial_{\mu} K \ldots K^{-1} \partial_{\sigma} K\right\}+\partial_{\mu} \Sigma_{\mu} .
$$

Integrating over all space finally yields

$$
\begin{gathered}
\int \mathrm{d}^{4} x \operatorname{Tr}\left(\delta A_{\mu} j_{\mu}\right)=\delta \int \mathrm{d}^{4} x\left\{\frac{1}{4} D_{1}-5 D_{2}+k\left(1+x^{2}\right)^{-2}\right. \\
\left.+\frac{1}{4} \int_{0}^{1} \mathrm{~d} t \epsilon_{\mu \nu \rho \sigma} \operatorname{Tr}\left[K^{-1} \partial_{t} K K^{-1} \partial_{\mu} K \ldots K^{-1} \partial_{\sigma} K\right]\right\}
\end{gathered}
$$

The term $k\left(1+x^{2}\right)^{-2}$ has been added to make the integral absolutely convergent.

To sum up, we have shown that

$$
\begin{aligned}
& \Gamma_{\text {reg }}-2 r \Gamma_{\text {reg }}^{0}=\alpha+\operatorname{Tr}(\ln N-\ln \hat{N}) \\
& +\frac{1}{48 \pi^{2}} \int \mathrm{d}^{4} x \ln \Omega \operatorname{Tr}\left(F_{\mu \nu} F_{\mu \nu}\right)+\frac{1}{24 \pi^{2}} \int \mathrm{d}^{4} x I_{1}(x) \\
& \quad+\frac{1}{24 \pi^{2}} \int \mathrm{d}^{4} x \int_{0}^{1} \mathrm{~d} t I_{2}(t, x) .
\end{aligned}
$$

Here, $\Gamma_{\text {reg }}^{0}$ equals $\Gamma_{\text {reg }}$ for the case of no background field and one color only, $\alpha$ is a number, which is constant on each connected component of the instanton manifold, and the integrands $I_{1}, I_{2}$ are given by

$$
\begin{aligned}
& I_{1}=\operatorname{Tr}\left\{f \partial_{\mu} f^{-1} f \partial_{\mu} f^{-1} f \partial_{\nu} f^{-1} f \partial_{\nu} f^{-1}\right\}-20 \operatorname{Tr}\left\{f^{2}\right\}+4 k\left(1+x^{2}\right)^{-2} \\
& I_{2}=\epsilon_{\mu \nu \rho \sigma} \operatorname{Tr}\left\{K^{-1} \partial_{t} K K^{-1} \partial_{\mu} K K^{-1} \partial_{\nu} K K^{-1} \partial_{\rho} K K^{-1} \partial_{\sigma} K\right\}
\end{aligned}
$$

\section{Computation of $\propto$}

Let $A_{\mu}$ be any $\mathrm{Sp}(r)$ instanton potential. Of course, $A_{\mu}$ can also be considered as an instanton configuration $\widetilde{A_{\mu}}$ in $\operatorname{Sp}(\tilde{r})$ for any $\widetilde{r} \geqslant r, v i z$, 


$$
\left.\tilde{A}_{\mu}=\begin{array}{|l|l|}
\hline A_{\mu} & 0 \\
\hline 0 & 0
\end{array}\right\}^{2 r}{ }_{2(\tilde{r}-r)}
$$

Denoting by $\widetilde{\Gamma}_{\text {reg }}$ the log of the determinant of the corresponding Dirac operator, we obviously have

$$
\widetilde{\Gamma}_{\text {reg }}-2 \widetilde{r} \Gamma_{\text {reg }}^{0}=\Gamma_{\text {reg }}-2 r \Gamma_{\text {reg }}^{0}
$$

i.e., the left-hand side of eq. (58) is invariant, when $A_{\mu}$ is imbedded into a higher group. The same holds for $N_{i j}, \hat{N}_{i j}$ and the integrals on the right-hand side of eq. (58) and must therefore be true for $\alpha$, too. Since any $k$-instanton solution can be imbedded or reduced to $\operatorname{Sp}(k)$, it follows that it is sufficient to compute $\alpha$ for $r=k$.

The instanton manifold is essentially a convex space for $r=k$ and is therefore connected ( $c f$. , the description of instantons given by Drinfeld and Manin [10]). $\alpha$ is hence the same for all $k$-instanton configurations. It can conveniently be computed by specializing eq. (58) to the case of a superposition of $k$ one-instanton solutions, each of them occupying one of the $k$ commuting SU(2) subgroups on the diagonal of $\operatorname{Sp}(k)$. Then, $\alpha=k \alpha_{1}$ where $\alpha_{1}$ is independent of $k$ and can be calculated for $k=1$.

The one-instanton solution of size $R$ centered at the origin has parameter matrices

$$
\begin{array}{ll}
a_{1}=R\left(\begin{array}{l}
1 \\
0 \\
0 \\
0
\end{array}\right), & a_{2}=R\left(\begin{array}{l}
0 \\
1 \\
0 \\
0
\end{array}\right), \\
b^{1}=\left(\begin{array}{l}
0 \\
0 \\
1 \\
0
\end{array}\right), & b^{2}=\left(\begin{array}{l}
0 \\
0 \\
0 \\
1
\end{array}\right) .
\end{array}
$$

Exploiting $\mathrm{O}(5)$ invariance, one finds that $T T^{+}$has the following two series of eigenvalues (e.g., ref. [12])

$$
\begin{aligned}
& E_{m}^{1}=4 R^{-2}(m+1)(m+4), \\
& d_{m}^{1}=\frac{1}{6}(m+2)(m+3)(2 m+5) ; \\
& E_{m}^{2}=4 R^{-2}(m+2)(m+3), \\
& d_{m}^{2}=\frac{1}{2}(m+1)(m+4)(2 m+5),
\end{aligned}
$$

( $E_{m}^{i}$ is the eigenvalue, $d_{m}^{i}$ its multiplicity and $m=0,1,2, \ldots$ ). In the vacuum case, there is only one series of eigenvalues

$$
\begin{aligned}
& E_{m}^{0}=4 R^{-2}(m+2)^{2}, \\
& d_{m}^{0}=\frac{2}{3}(m+1)(m+2)(m+3) .
\end{aligned}
$$


$\Gamma_{\text {reg }}$ for the one instanton solution (63) can now be obtained using 't Hooft's method [13] to compute the regularized sum (39) over all eigenvalues $(64 a, b)$ :

$$
\begin{aligned}
\Gamma_{\mathrm{reg}} & =\sum_{i=1}^{\nu} e_{i} \ln \left(M_{i} R\right)\left[\frac{1}{24}\left(M_{i} R\right)^{4}+\frac{1}{3}\left(M_{i} R\right)^{2}+\frac{41}{45}\right]+\ln R \\
& -\frac{4}{3} \zeta^{\prime}(-1)-\frac{8}{3} \zeta^{\prime}(-3)-\frac{41}{45} \ln 2-\ln 3+\frac{221}{540}
\end{aligned}
$$

$(\zeta(z)$ is Riemann's zeta function, ref. [14], subsect. 9.5). Similarly, from the series (64c) we get

$$
\begin{aligned}
& \Gamma_{\mathrm{reg}}^{0}=\sum_{i=1}^{\nu} e_{i} \ln \left(M_{i} R\right)\left[\frac{1}{48}\left(M_{i} R\right)^{4}+\frac{1}{6}\left(M_{i} R\right)^{2}+\frac{11}{90}\right] \\
& \quad+\frac{4}{3} \zeta^{\prime}(-1)-\frac{4}{3} \zeta^{\prime}(-3)-\frac{11}{90} \ln 2-\frac{1}{270} .
\end{aligned}
$$

The left-hand side of eq. (58) thus becomes

$$
\Gamma_{\text {reg }}-2 \Gamma_{\text {reg }}^{0}=\frac{2}{3} \sum_{i=1}^{\nu} e_{i} \ln M_{i}+\frac{1}{3} \ln R-4 \zeta^{\prime}(-1)-\frac{2}{3} \ln 2-\ln 3+\frac{5}{12} .
$$

It is a trivial matter to evaluate the right-hand side of eq. (58) for the special configuration (63). Comparing with eq. (67), we then obtain $\alpha_{1}$ and hence $\alpha$ :

$$
\alpha=k\left\{\frac{2}{3} \sum_{i=1}^{\nu} e_{i} \ln M_{i}-4 \zeta^{\prime}(-1)-\ln 2+\frac{5}{12}\right\} .
$$

\section{Summary and discussion}

Our final formula for the regularized determinant (38) of the Dirac operator $D$ (eq. (23)) in a general $\mathrm{Sp}(r) k$-instanton solution is

$$
\begin{gathered}
\Gamma_{\mathrm{reg}}-2 r \Gamma_{\mathrm{reg}}^{0}=k\left\{\frac{2}{3} \sum_{i=1}^{\nu} e_{i} \ln M_{i}-4 \zeta^{\prime}(-1)-\ln 2+\frac{5}{12}\right\} \\
+\operatorname{Tr}(\ln N-\ln \hat{N})+\frac{1}{48 \pi^{2}} \int \mathrm{d}^{4} x \ln \Omega \operatorname{Tr}\left(F_{\mu \nu} F_{\mu \nu}\right) \\
+\frac{1}{24 \pi^{2}} \int \mathrm{d}^{4} x I_{1}(x)+\frac{1}{24 \pi^{2}} \int \mathrm{d}^{4} x \int_{0}^{1} \mathrm{~d} t I_{2}(t, x) .
\end{gathered}
$$

Here, $\Gamma_{\text {reg }}$ is the determinant with vanishing background field, the $k \times k$ matrices $N$ and $\tilde{N}$ (eq. (47)) are built from the zero modes (27) of $D$, and the integrands $I_{1}$, 
$I_{2}$ are

$$
\begin{aligned}
I_{1} & =\operatorname{Tr}\left\{f \partial_{\mu} f^{-1} f \partial_{\mu} f^{-1} f \partial_{\nu} f^{-1} f \partial_{\nu} f^{-1}\right\} \\
& -5 \operatorname{Tr}\left\{f b_{A}^{+} b^{A} f b_{B}^{+} b^{B}\right\}+4 k\left(1+x^{2}\right)^{-2}, \\
I_{2} & =\epsilon_{\mu \nu \rho \sigma} \operatorname{Tr}\left\{K^{-1} \partial_{t} K K^{-1} \partial_{\mu} K K^{-1} \partial_{\nu} K K^{-1} \partial_{\rho} K K^{-1} \partial_{\sigma} K\right\} .
\end{aligned}
$$

The real symmetric $k \times k$ matrix $f(x)$ is defined in terms of the instanton parameters by eq. (15) and, finally,

$$
K(t, x)_{i j}=(1-t)\left(1+x^{2}\right) \frac{1}{2}\left(b_{A}^{+} b^{A}\right)_{i j}+t f^{-1}(x)_{i j}
$$

Eq. (69) simplifies in the infinite volume limit $R=\infty$ :

$$
\begin{aligned}
& \lim _{R \rightarrow \infty}\left(\Gamma_{\mathrm{reg}}-2 r \Gamma_{\mathrm{reg}}^{0}\right)=k\left\{\frac{2}{3} \sum_{i=1}^{\nu} e_{i} \ln M_{i}-4 \zeta^{\prime}(-1)-\frac{2}{3} \ln 2+\frac{5}{12}\right\} \\
& +\frac{1}{24 \pi^{2}} \int \mathrm{d}^{4} x I_{1}(x)+\frac{1}{24 \pi^{2}} \int \mathrm{d}^{4} x \int_{0}^{1} \mathrm{~d} t I_{2}(t, x) .
\end{aligned}
$$

This is a tractable expression, although it is not completely explicit. Maybe, with sufficient ingenuity, one could calculate the integrals, but we did not attempt to do so. The integrand $I_{2}$ vanishes for all one- and two-instanton solutions. We checked that in general it contributes, when $k \geqslant 3$, thus indicating that two-instanton solutions are not generic multi-instanton configurations. $I_{2}$ also vanishes for 't Hooft's instanton solutions

$$
A_{\mu}=\frac{1}{2} \bar{\sigma}_{\mu \nu} \partial_{\nu} \ln \rho, \quad \rho=1+\sum_{i=1}^{k} \frac{\lambda_{i}^{2}}{\left(x-a^{i}\right)^{2}},
$$

which are therefore rather special, too. For these solutions, eq. (71) can be written more compactly:

$$
\begin{aligned}
& \lim _{R \rightarrow \infty}\left(\Gamma_{\text {reg }}-2 r \Gamma_{\text {reg }}^{0}\right)=k\left\{\frac{2}{3} \sum_{i=1}^{\nu} e_{i} \ln M_{i}-4 \xi^{\prime}(-1)-\frac{2}{3} \ln 2\right\} \\
& +\frac{1}{96 \pi^{2}} \int \mathrm{d}^{4} x \ln \tilde{\rho} \square^{2} \ln \tilde{\rho}+\frac{2}{3} \sum_{i=1}^{k} \ln \lambda_{i}+\frac{1}{3} \sum_{i<j} \ln \left(a^{i}-a^{j}\right)^{2},
\end{aligned}
$$

where, following Belavin et al. [3] ${ }^{\star}, \tilde{\rho}$ is defined by

$$
\tilde{\rho}=\rho \prod_{i=1}^{k}\left(x-a^{i}\right)^{2} .
$$

\footnotetext{
* Our result, however, seems to disagree with their eq. (7).
} 
An equivalent form of eq. (73) has previously been found by Brown and Creamer [7].

The formulae for the determinants, which we have derived here, are strikingly similar to the corresponding ones in two-dimensional non-linear $\sigma$-models $[1,2]$. This suggests that the integrals over $I_{1}$ and $I_{2}$ have perhaps a universal geometrical meaning relating to the topological and metric properties of the instanton manifold.

To compute the determinant of the gluon fluctuation operator via supersymmetry, requires the quarks to be in the adjoint rather than the fundamental representation of the gauge group. In view of the tensor product formula for instantons [15], however, our result applies to this case as well. The ensuing exact instanton gas will be rather complicated and what its physical properties are, remains to be seen.

One of us (B.B.) thanks H. Krasemann and Z. Kunszt for discussions about computer problems.

\section{Appendix}

Short-distance expansion of $S_{-}^{M}(x, y)_{A A^{\prime}}$

$S_{-}^{M}$ is defined by

$$
S_{-}^{M}(x, y)_{A A^{\prime}}=2 \Omega(x)^{-1 / 2} i\left(e_{\mu}\right)_{A B^{\prime}} D_{\mu} G^{M}(x, y)^{B^{\prime}{ }_{A^{\prime}}}
$$

where $G^{M}$ is the Green function for $T T^{+}+M^{2}$ :

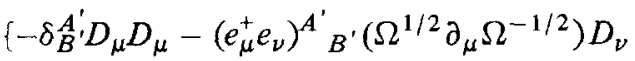

$$
\begin{aligned}
& \left.+\frac{1}{4} \Omega M^{2} \delta_{B^{\prime}}^{A^{\prime}}\right\} G^{M}(x, y)^{B^{\prime}} C^{\prime}=\frac{1}{4} \Omega^{1 / 2} \delta(x-y) .
\end{aligned}
$$

We first derive the short-distance expansion of $G^{M}(x, y)$ for $y=0$ and shall later exploit $\mathrm{O}(5)$ covariance to extend the result to all $y$. Defining

$$
\begin{aligned}
& U_{\mu}=2 A_{\mu}+e_{\nu}^{+} e_{\mu}\left(\Omega^{1 / 2} \partial_{\nu} \Omega^{-1 / 2}\right), \\
& V=\partial_{\mu} A_{\mu}+A_{\mu} A_{\mu}+e_{\mu}^{+} e_{\nu}\left(\Omega^{1 / 2} \partial_{\mu} \Omega^{-1 / 2}\right) A_{\nu}, \\
& \kappa=1-\frac{1}{4} \Omega, \\
& W=U_{\mu} \partial_{\mu}+V+\kappa M^{2},
\end{aligned}
$$

eq. (A.2) reads (spinorial indices are suppressed)

$$
\left\{-\partial_{\mu} \partial_{\mu}+M^{2}-W\right\} G^{M}(x, 0)=\frac{1}{2} \delta(x) \text {. }
$$

The perturbative solution of this equation is

$$
\begin{gathered}
G^{M}(x, 0)=\frac{1}{2} \sum_{m=0}^{\infty} \int \mathrm{d}^{4} x_{1} \ldots \mathrm{d}^{4} x_{m} G_{0}\left(x-x_{1}\right) \\
\times W\left(x_{1}\right) G_{0}\left(x_{1}-x_{2}\right) \ldots W\left(x_{m}\right) G_{0}\left(x_{m}\right),
\end{gathered}
$$


where the free propagator is

$$
G_{0}(z)=\int \frac{\mathrm{d}^{4} p}{(2 \pi)^{4}} \mathrm{e}^{i p z}\left(p^{2}+M^{2}\right)^{-1} .
$$

Each term in the sum (A.4) can be expanded for $x \rightarrow 0$. It is sufficient to calculate the expansion up to and including the order $x_{\mu}$. Define

$$
C_{m}(x)=\int \mathrm{d}^{4} x_{1} \ldots \mathrm{d}^{4} x_{m} G_{0}\left(x-x_{1}\right) W\left(x_{1}\right) \ldots W\left(x_{m}\right) G_{0}\left(x_{m}\right) .
$$

Expanding

$$
W(z)=W^{0}+z_{\mu} W_{\mu}^{1}+z_{\mu} z_{\nu} W_{\mu \nu}^{2}+\ldots,
$$

the integral (A.6) can be evaluated in p-space and reduces to a sum of Bessel functions. Most of the terms, however, do not contribute in the limit where first $x \rightarrow 0$ and then $M \rightarrow \infty$. In particular, for $m \geqslant 4, C_{m}(x)$ is once continuously differentiable at $x=0$ and $C_{m}(0)$ as well as $\partial_{\mu} C_{m}(0)$ vanish for $M \rightarrow \infty$. Also, integrals involving $W^{l}, l \geqslant 4$, need not be considered for the same reason. Thus, we are left with a finite number of integrals to compute and expand for $x \rightarrow 0, M \rightarrow \infty$. This calculation is lengthy but straightforward and we therefore merely quote the result:

$$
\sum_{j=1}^{\nu} e_{j} S_{-}^{M_{j}}(x, 0)_{A A^{\prime}}=-\frac{1}{2} i\left(e_{\mu}\right)_{A A^{\prime}} D_{\mu}\left[U(x, 0)\left(4 \pi^{2} x^{2}\right)^{-1}\right] .
$$

$U(x, 0)$ is the gauge parallel transporter from the origin to $x$ along the straight line connecting the two (eq. (42)).

The $O(5)$ covariant expression reducing to (A.8) for $y=0$ is

$$
\begin{aligned}
& \sum_{j=1}^{\nu} e_{j} S_{-}^{M_{j}}(x, y)_{A A^{\prime}}=-\frac{1}{2} i \Omega(x)^{1 / 4}\left(e_{\mu} V^{\mathrm{g}}(x, y)\right)_{A A^{\prime}} \\
& \quad \times D_{\mu}\left\{U ^ { \mathrm { g } } ( x , y ) \left[\left(4 \pi^{2} \Omega(x)^{1 / 2}(x-y)^{2} \Omega(y)^{1 / 2}\right)^{-1}\right.\right. \\
& \left.\left.\quad-\left(32 \pi^{2} R^{2}\right)^{-1} \ln \left(\Omega(x)^{1 / 2}(x-y)^{2} \Omega(y)^{1 / 2}\right)\right]\right\} \Omega(y)^{3 / 4} .
\end{aligned}
$$

Here, $U^{\mathrm{g}}(x, y)$ is the gauge parallel transporter along the (shorter) geodesic connecting $y$ with $x$. Similarly, $V^{\mathrm{g}}(x, y)$ is the spin parallel transporter along the same geodesic,

$$
V^{\mathrm{g}}(x, y)=\mathrm{P} \exp \left\{-\int_{y}^{x} \mathrm{~d} z_{\mu} \bar{\sigma}_{\mu \nu} \frac{1}{4} \partial_{\nu} \ln \Omega\right\},
$$

where $\bar{\sigma}_{\mu \nu}=\frac{1}{2}\left(e_{\mu}^{+} e_{\nu}-e_{\nu}^{+} e_{\mu}\right)$. Noting that

$$
U^{\mathrm{g}}(x, y)=U(x, y)+(x-y)^{2}(x-y)_{\mu} F_{\mu \nu}(y) y_{\nu} \frac{\Omega(y)^{1 / 2}}{12 R^{2}}+\mathrm{O}\left((x-y)^{4}\right)
$$


(and an analogous formula for $V^{g}(x, y)$ ), another tedious but straightforward computation shows that eq. (A.9) is equivalent to eq. (43) up to terms vanishing at $x=y$.

\section{References}

[1] V.A. Fateev, I.V. Frolov and A.S. Schwarz, Nucl. Phys. B154 (1979) 1.

[2] B. Berg and M. Lüscher, DESY 79/17, Comm. Math. Phys., to appear.

[3] A.A. Belavin, V.A. Fateev, A.S. Schwarz and Yu.S. Tyupkin, Phys. Lett. 83B (1979) 317.

[4] A. D'Adda and P. Di Vecchia, Phys. Lett. 73B (1978) 162.

[5] E.F. Corrigan, D.B. Fairlie, P. Goddard and S. Templeton, Nucl. Phys. B140 (1978) 31.

[6] N.H. Christ, E.J. Weinberg and N.K. Stanton, Phys. Rev. D18 (1978) 2013.

[7] L.S. Brown and D.B. Creamer, Phys. Rev. D18 (1978) 3695.

[8] G. 't Hooft, unpublished.

[9] M.F. Atiyah, N.J. Hitchin, V.G. Drinfeld and Yu.I. Manin, Phys. Lett. 65A (1978) 185.

[10] V.G. Drinfeld and Yu.I. Manin, Comm. Math. Phys. 63 (1978) 177.

[11] H. Osborn, Nucl. Phys. B140 (1978) 45.

[12] S. Chadha, A. D'Adda, P. Di Vecchia and F. Nicodemi, Phys. Lett. 67B (1977) 470; 72B (1977) 103.

[13] G. 't Hooft, Phys. Rev. D14 (1976) 3432 (E: D18 (1978) 2199).

[14] I.S. Gradshteyn and I.W. Ryzhik, Table of integrals, series and products (Academic Press, New York, 1965).

[15] E.F. Corrigan, P. Goddard and S. Templeton, Nucl. Phys. B151 (1979) 93; V.G. Drinfeld and Yu.I. Manin, Yad. Fiz. 29 (1979) 1646. 